\title{
A Ratio Scale Metric and the Compatibility of Ratio Scales: On the Possibility of Arrow's Impossibility Theorem
}

\author{
Thomas L. Saaty \\ 322 Mervis Hall, University of Pittsburgh \\ Pittsburgh, PA 15260 , U.S.A
}

\begin{abstract}
We develop a metric for ratio scales and explore the notion of compatibility of two sets of measurements of a set of objects or properties on a ratio scale. We briefly address Arrow's impossibility theorem. We maintain that it is not as impossible as claimed when, as in reality, a certain degree of inconsistency and incompatibility are allowed.
\end{abstract}

Keywords: ratio scale, metric, compatibility, impossibility, group decision making, diagnosis

\section{INTRODUCTION [1]}

A scale is a triple: a set of objects or properties, a set of numbers and a mapping from the object's to the numbers. If the number of objects is $n$, the scale associated with them is an n-vector. A set of numbers belongs to a ratio scale if it is invariant under a similarity transformation (multiplication by a positive number). It follows that all the points on a ray from an origin in $R_{+}^{n}$ belong to the same ratio scale. Each point on the ray is an n-tuple. A problem that often arises in practice is: Given two n-tuples $w=\left(w_{1}, \ldots, w_{n}\right)$ and $u=\left(u_{1}, \ldots, u_{n}\right)$, which represent two sets of measurements on a ratio scale of the same set of alternatives on a single property how close are these measurements? A second problem is that we have known standard measurements of the symptoms of a disease on several properties. These single readings on each property âre given by an $n$-tuple $p=\left(p_{l}, \ldots, p_{n}\right)$ and a patient arrives whose readings are $q=$ $\left(q_{1}, \ldots, q_{n}\right)$, how do we decide whether the patient is likely to have the disease? Examples of two metrics which have been used to determine closeness on the same property are the Euclidean metric $\left(\sum_{i=1}^{n}\left(w_{i}-u_{i}\right)^{2}\right)^{1 / 2}$ and the Hilbert metric $\log \left[\max \left(w_{i} / u_{i}\right) / \min \left(w_{i} / u_{i}\right)\right]$. The first of these metrics ignores that $w$ and $u$ belong to a ratio scale and the second ignores all but the maximum and minimum values. We have tested both these metrics and neither has been found to be sufficiently sensitive to enable a clear decision. What we need to consider is all the numbers and their ratios. In performing fine measurements one can hardly object to seeking a thorough metric based on all the ratios.

This note examines the idea of deriving a valuation index or metric for a better comparison of numbers when they belong to a ratio scale than we have so far.

\section{A RATIO SCALE METRIC - THE COMPATIBILITY OF MEASUREMENTS}

Given the vector $w=\left(w_{l}, \ldots, w_{n}\right)$ where all the $w_{i}$ belong to the same scale, we consider the matrix of all possible ratios $A=\left(a_{i j}\right)=\left(w_{i} / w_{j}\right)$. This matrix is reciprocal, that is $a_{j i}=1 / a_{i j}$. The Hadamard product of a reciprocal matrix $A$ and its transpose $A^{T}$ is given by 


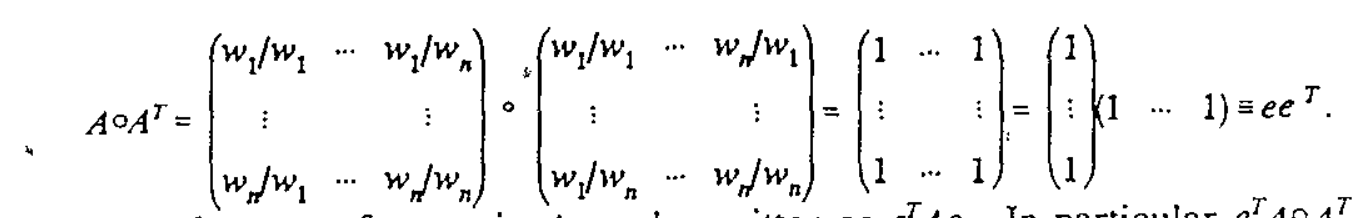

The sum of the elements of a matrix $A$ can be written as $e^{T} A e$. In particular $e^{T} A^{\circ} A^{T} e=n^{2}$.

If we assume that two vectors $w=\left(w_{1}, \ldots, w_{n}\right)$ and $u=\left(u_{1}, \ldots, u_{n}\right)$, whose coordinates are measured on the same ratio scale, differ by a perturbation matrix $E=\left(\epsilon_{i j}\right)$ so that $\frac{u_{1}}{u_{j}}=\frac{u_{i}}{w_{j}} \varepsilon_{i}$ and if their corresponding matrices are $A=\left(\frac{w_{i}}{w_{j}}\right)$ and $B=\left(\frac{u_{i}}{u_{j}}\right)$, the Hadamard product we want is $A^{\circ} B^{T}=\left(\varepsilon_{i j}\right)$. We are concerned with the closeness of $e^{T} A^{\circ} B^{T} e=\sum_{i, j=1}^{n} \frac{w_{i}}{w_{j}} \frac{u_{j}}{u_{i}}$ to its minimum value $n^{2}$ or with the closeness of the normalized vectors $w_{i} / \sum_{i=1}^{n} w_{i}$ and $u_{i} / \sum_{i=1}^{n} u_{i}$ to each other. It is easy to prove the first three theorems below.

Theorem 1: If $A=\left(\frac{w_{i}}{w_{j}}\right)$ then $A=v w, v=\left(\frac{1}{w_{1}}, \ldots, \frac{1}{w_{n}}\right)^{T}, w=\left(w_{1}, \ldots, w_{n}\right)$

Corollary: If $\sum_{i=1}^{n} w_{i}=1$ then $e^{T} A e=e^{T_{v}}$

Theorem 2: $e^{T} A e=e^{T}$ vwe $=\sum_{j=1}^{n} \frac{1}{w_{j}} \cdot \sum_{i=1}^{n} w_{i}$.

We define compatibility between two ratio scales $w$ and $u$ as $c(w, u)=e^{T} A \circ B^{T} e$.

Ratio scales have no zero value but only a zero origin. The following theorem is analogous to the first axiom of a metric.

Theorem 3: $c(w, u)=n^{2}$ if and only if $w=u$

Proof: If $A=B$ then $A^{T}=B^{T}$ and $e^{T} A{ }^{\circ} B^{T} e=n^{2}$ or $c(w, u)=n^{2}$. Conversely, assume that $c(w, u)$ $=n^{2}$. The sum of the elements of the reciprocal matrix $A \circ B^{T}$ can be represented in pairs of terms of the convex form $x+1 / x$ each of which has a minimum value of 2 . Since the sum of the elements is equal to $n^{2}$, each term $x+1 / x$ must be equal to 2 which is attained if and only if $x=1$. If we let $x=\frac{w_{i}}{w_{j}} \frac{u_{j}}{u_{i}}$, it follows that $\frac{w_{i}}{w_{j}}=\frac{u_{i}}{u_{j}}$ for all $i$ and $j$ and hence $w=u$.

A special case of this theorem is that of $n=1$, in other words the comparison of two ratio scales rests on the comparison of a single measurement from each. If we define $d(w, u)=$ $\log c(w, u)$ we obtain the first axiom of an ordinary metric in geometry.

One can generate a relative ratio scale when a set of attributes are being compared in a test. What should one do when there is only one attribute and how do we compare two measurements of the same attribute. There are two ways to obtain measurement for a single attribute. One is through relative comparisons with a known ideal state of that attribute obtained from memory. This is the only way to create measurement when one deals with an intangible 
attribute. The other, useful mostly for physical measurement, is to create a scale with a unit for measuring that attribute. It is a special case of relative measurement. Physical measurement is related to the idea of distance and more abstractly to a metric and geometry. Measurement on a physical scale may or may not, belong to a ratio scale. If it does, one can sometimes directly form ratios of such measurements on such a scale or alternatively use the measurements to develop relative measurements or create new ratios from them through judgment. Alternatively one can take the differences of two measurements on a difference scale each of whose readings is itself is the logarithm of a ratio scale measurement. These are the two possible ways to create physical measurement that is compatible with the fundamental process of relative measurement.

One may speculate that if reciprocal comparisons are so intrinsic to the brain. the geometric structure based on them should in principle be the most general to include all known geometries las a special case because we can only understand geometries using understanding fundamentally based on paired comparisons.

Lemma: $\sum_{i=1}^{n} a_{i} b_{i} \leq\left(\sum_{i=1}^{n} a_{i}\right)\left(\sum_{i=1}^{n} b_{i}\right) \quad a_{i}, b_{i} \geq 0, \quad i=1, \ldots, n$.

Proof: Note that each term on the right is positive and that the left side is included in the right side.

Theorem 4: $c(w, v) \leq c(w, u) c(u, v)$

Proof: $e^{T} A^{\circ} \circ C^{T} e=e^{T} A \circ B^{T} \circ B \circ C^{T} e \leq e^{T} A \circ B^{T} e e^{T} B \circ C^{T} e=c(w, u) c(u, v)$ having used the lemma.

We note that if we have to compare a single reading from a ratio scale with a standard value on the same scale we simply take their ratio for the Hadamard product of their two single element matrices. Thus if one reading is $p$ and the other is $q$ we have $c(p, q)=p / q$ and .

$$
d(p, q)=\log c(p, q)=\log p / q \text {. }
$$

If $P$ and $Q$ are vectors defined by a set of coordinates in cartesian space, we need to use one of the many possible norms for that vector to form the ratio $\mathrm{p} / \mathrm{g}$.

The transformation $d(w, u)=\log c(w, u)$ for $n=1$ satisfies the two axioms of a metric 1) given in Theorem 3 with $n=1$ and 2) given in Theorem 4 . In addition, it is easy to show that 3) $d(w, u)=d(u, w), 4) d(w, u)$ is a continuous function of $w$ and $u, 5)$ if $u$ lies on a line between $w$ and $v$ then $d(w, v)=d(w, u)+d(w, v)$ (the geodesic property), and 6) $d(\alpha w, \alpha u)=$ $d(w, u), \alpha>0$, for all $w$ and $u$ (invariance with respect to the ratio scale property). A second metric $d^{\prime}(w, u)=k d(w, u)$ for some $\mathrm{k}>0$ also satisfies all these conditions. The space of all $w$ 's and $u$ 'siendowed with the metric $d(w, u)$ is a hyperbolic space [2].

What is a good bound to place on compatibility as defined by $e^{T} A^{\circ} B^{T} e$ ? From dealing with the measurement of tolerable inconsistency we have developed the argument that one can tolerate perturbations that are one order of magnitude or less when compared with the original number. Perturbations that are as large (of the same order of magnitude) as the number itself are unacceptable. Thus an admissible bound for compatibility can be set at 1.100 . It is in accord with the idea that a $10 \%$ deviation is at the upper end of acceptability. 


\section{COMPATIBILITY AND CONSISTENCY}

Consistency is concerned with the compatibility of a matrix of the ratios constructed from a principal right eigenvector with the matrix of judgments from which it is derived. Compatibility is concerned with two different vectors. If the matrix of judgments is inconsistent. is it compatible with the matrix of eigenvector ratios? The following theorem and the table following it show that there is a relation between consistency and compatibility. Comparison of the two indices suggests that for the cases of 3-5 alternatives the Compatibility Index should have a smaller value than 1.1 .

Let $W=\left(w_{i} / w_{j}\right)$ be the matrix of ratios of the principal right eigenvector $w=\left(w_{l}^{\prime}, \ldots, w_{r}^{\prime}\right)$ of the positive reciprocal matrix $A$ and $\lambda_{\max }$ be the corresponding principal eigenvector and let $\sum_{i=1}^{n} w_{i}=1$. We define the Compatibility Index (S.I.) of a matrix of judgments and the matrix of derived eigenvector ratios as S.I. $=\frac{1}{n^{2}} e^{T} A \circ W^{T} e$.

Theorem 5: $\frac{1}{n^{2}} e^{T} A \circ W^{T} e=\frac{\lambda_{\max }}{n}$

Proof: From $A w=\lambda_{\max } w$ we have

and

$$
\sum_{j=1}^{n} a_{i j} w_{j}=\lambda_{\max } w_{i}
$$

$$
\frac{1}{n^{2}} e^{T} A^{\circ} w^{T_{e}}=\frac{1}{n^{2}} \sum_{i=1}^{n} \sum_{j=1}^{n} a_{i j} \frac{w_{j}}{w_{i}}=\frac{\lambda_{\max }}{n}
$$

\begin{tabular}{cccccc}
\hline $\begin{array}{c}\text { Number of } \\
\text { alternatives }(\mathrm{n})\end{array}$ & $\begin{array}{c}\text { Compatibility } \\
\text { Index (S.I.) }\end{array}$ & $\lambda_{\max }$ & C.I. $=\frac{\lambda_{\max }-n}{n-1}$ & R.I. & C.R. $=\frac{C . I .}{R . I .}$ \\
\hline 3 & 1.017 & 3.052 & 0.026 & 0.52 & 0.05 \\
4 & 1.053 & 4.214 & 0.071 & 0.89 & .0 .08 \\
5 & 1.089 & 5.444 & 0.111 & 1.11 & 0.10 \\
6 & 1.104 & 6.625 & 0.125 & 1.25 & 0.10 \\
7 & 1.116 & 7.810 & 0.135 & 1.35 & 0.10 \\
8 & 1.123 & 8.980 & 0.140 & 1.40 & 0.10 \\
9 & 1.129 & 10.160 & 0.145 & 1.45 & 0.10 \\
10 & 1.134 & 11.341 & 0.149 & 1.49 & 0.10 \\
11 & 1.137 & 12.510 & 0.151 & 1.51 & 0.10 \\
12 & 1.141 & 13.694 & 0.154 & 1.54 & 0.10 \\
13 & 1.144 & 14.872 & 0.156 & 1.56 & 0.10 \\
14 & 1.146 & 16.041 & 0.157 & 1.57 & 0.10 \\
15 & 1.147 & 17.212 & 0.158 & 1.58 & 0.10 \\
\hline
\end{tabular}




\section{EXAMPLES}

Consider the Hadamard product:

We have

$$
\begin{gathered}
\left(\begin{array}{ccc}
1 & 2 & 4 \\
1 / 2 & 1 & 2 \\
1 / 4 & 1 / 2 & 1
\end{array}\right] \circ\left[\begin{array}{ccc}
1 & 1 / 3 & 1 / 5 \\
3 & 1 & 3 / 5 \\
5 & 5 / 3 & 1
\end{array}\right]=\left[\begin{array}{ccc}
1 & 2 / 3 & 4 / 5 \\
3 / 2 & 1 & 6 / 5 \\
5 / 4 & 5 / 6 & 1
\end{array}\right) \\
\frac{1}{n^{2}} e^{T} A^{\circ} B^{T} e=\frac{9}{9}=1.028
\end{gathered}
$$

The ratio scale vectors corresponding to the two matrices are $\left[\frac{4}{7}, \frac{2}{7}, \frac{1}{7}\right]^{T}$ and $\left[\frac{3}{4.6}, \frac{1}{4.6}, \frac{.6}{6.6}\right]^{T}$ which by this measure are considered close.

Again $\quad\left[\begin{array}{ccc}1 & 2 & 4 \\ 1 / 2 & 1 & 2 \\ 1 / 4 & 1 / 2 & 1\end{array}\right] \circ\left[\begin{array}{ccc}1 & 1 / 3 & 1 / 9 \\ 3 & 1 & 1 / 3 \\ 9 & 3 & 1\end{array}\right]=\left[\begin{array}{ccc}1 & 2 / 3 & 4 / 9 \\ 3 / 2 & 1 & 2 / 3 \\ 9 / 4 & 3 / 2 & 1\end{array}\right)$

from which we have $\quad \frac{1}{n^{2}} e^{T} A^{\circ} B^{T} e=\frac{10 \frac{1}{36}}{9}=1.114$

The ratio scale vectors in this case are $\left[\frac{4}{7}, \frac{2}{7}, \frac{1}{7}\right]^{T}$ and $\left[\frac{9}{13}, \frac{3}{13}, \frac{1}{13}\right]^{T}$ whose closeness may be considered as a borderline case.

An example of a large perturbation of a matrix is:

from which we have

$$
\begin{aligned}
\left(\begin{array}{ccc}
1 & 2 & 4 \\
1 / 2 & 1 & 2 \\
1 / 4 & 1 / 2 & 1
\end{array}\right) \circ\left(\begin{array}{ccc}
1 & 1 / 9 & 1 \\
9 & 1 & 9 \\
1 & 1 / 9 & 1
\end{array}\right) & =\left(\begin{array}{ccc}
1 & 2 / 9 & 4 \\
9 / 2 & 1 & 18 \\
1 / 4 & 1 / 18 & 1
\end{array}\right) \\
e^{T} A \circ B^{T} e & =\frac{30.027}{9}=3.336
\end{aligned}
$$

The ratio scale vectors of the two matrices are respectively

which by any measure are not close. We also note that from

$$
\left(\frac{4}{7}, \frac{2}{7}, \frac{1}{7}\right)^{T} \text { and }\left(\frac{1}{11}, \frac{9}{11}, \frac{1}{11}\right)^{T}
$$

$$
\left[\begin{array}{ccc}
1 & 2 & 4 \\
1 / 2 & 1 & 2 \\
1 / 4 & 1 / 2 & 1
\end{array}\right) \circ\left(\begin{array}{ccc}
1 & 2 & 4 \\
1 / 2 & 1 & 2 \\
1 / 4 & 1 / 2 & 1
\end{array}\right)=\left(\begin{array}{ccc}
1 & 4 & 16 \\
1 / 4 & 1 & 4 \\
1 / 16 & 1 / 4 & 1
\end{array}\right)
$$


we have

$$
e^{T} A \circ B^{T} e=\frac{27 \frac{9}{16}}{9}=3.063
$$

The two vectors of $\mathrm{A}$ and $\mathrm{B}$ are

which are not close.

$$
\left(\frac{4}{7}, \frac{2}{7}, \frac{1}{7}\right)^{T} \text { and }\left(\frac{1}{7}, \frac{2}{7}, \frac{4}{7}\right)^{T}
$$

Finally, consider the following two cases of $4 \times 4$ matrices constructed to iest compatibility with the vector $\left[\begin{array}{llll}.05 & .15 & .30 & .50\end{array}\right]^{\mathrm{T}}$ of:

(a) once to a close vector $\left[\begin{array}{llll}.08 & .22 & .25 & .45\end{array}\right]^{\mathrm{T}}$

$$
\left[\begin{array}{cccc}
1 & \frac{.05}{.15} & \frac{.05}{.30} & \frac{.05}{.50} \\
\frac{.15}{.05} & 1 & \frac{.15}{.30} & \frac{.15}{.50} \\
\frac{.30}{.05} & \frac{.30}{.15} & 1 & \frac{.30}{.50} \\
\frac{.50}{.05} & \frac{.50}{.15} & \frac{.50}{.30} & 1
\end{array}\right) \circ\left[\begin{array}{cccc}
1 & \frac{.22}{.08} & \frac{.25}{.08} & \frac{.45}{.08} \\
\frac{.08}{.22} & 1 & \frac{.25}{.22} & \frac{.45}{.22} \\
\frac{.08}{.25} & \frac{.22}{.25} & 1 & \frac{.45}{.25} \\
\frac{.08}{.45} & \frac{.22}{.45} & \frac{.25}{.45} & 1
\end{array}\right]=\left[\begin{array}{lllll}
1.00 & 0.92 & 0.52 & 0.56 \\
1.09 & 1.00 & 0.57 & 0.61 \\
1.92 & 1.76 & 1.00 & 1.08 \\
1.77 & 1.63 & 0.93 & 1.00
\end{array}\right)
$$

with

$$
e^{T} A \circ B^{T} e=\frac{17.36}{16}=1.085
$$

which is tolerable; and

(b) once to a not so close vector $\left[\begin{array}{llll}.03 & .25 & .10 & .62\end{array}\right]^{\mathrm{T}}$.

and

$$
\begin{gathered}
{\left[\begin{array}{cccc}
1 & \frac{.05}{.15} & \frac{.05}{.30} & \frac{.05}{.50} \\
\frac{.15}{.25} & 1 & \frac{.15}{.30} & \frac{.15}{.50} \\
\frac{.30}{.05} & \frac{.30}{.15} & 1 & \frac{.30}{.50} \\
\frac{.50}{.05} & \frac{.50}{.15} & \frac{.50}{.30} & 1
\end{array}\right] \circ\left[\begin{array}{cccc}
1 & \frac{.25}{.03} & \frac{.10}{.03} & \frac{.62}{.03} \\
\frac{.03}{.25} & 1 & \frac{.10}{.25} & \frac{.62}{.25} \\
\frac{.03}{.10} & \frac{.25}{.10} & 1 & \frac{.62}{.10} \\
\frac{.03}{.62} & \frac{.25}{.62} & \frac{.10}{.62} & 1
\end{array}\right]=\left[\begin{array}{lllll}
1.00 & 2.77 & 0.55 & 2.07 \\
0.36 & 1.00 & 0.20 & 0.74 \\
1.80 & 5.00 & 1.00 & 3.72 \\
0.48 & 1.34 & 0.27 & 1.00
\end{array}\right]} \\
e^{T} A^{\circ} B^{T} e=\frac{22.99}{16}=1.437
\end{gathered}
$$

which is not tolerable.

\section{THE CASE OF SEVERAL RATIO SCALES}

Consider now the case of two vectors $p=\left(p_{1}, \ldots, p_{n}\right)$ and $q=\left(q_{l}, \ldots, q_{n}\right)$ each of which consists of single readings each on one of $n$ scales as in the case of a patient who takes several tests measured in different ways. How should we judge how close are $p$ and $q$ ? Here 
we first consider the ratios $\frac{p_{i}}{q_{i}}$. We consider the sum $\frac{1}{2 n} \sum_{i=1}^{n}\left(\frac{p_{i}}{q_{i}}+\frac{q_{i}}{p_{i}}\right)$ and require that it be close to one. If on the other hand we can determine through paired comparisons that the properties have different priorities given by a normalized vector $\alpha=\left(\alpha_{1}, \ldots, \alpha_{n}\right)$, then we require that $\frac{1}{2} \sum_{i=1}^{n} \alpha\left(\frac{p_{i}}{q_{i}}+\frac{q_{i}}{p_{i}}\right)$ be close to one. If we have multiple readings on each property, we could use the same analysis for each as in the previous discussion for a single attribute and add the outcomes and require the total not to exceed 1.10. For a mixed vector with several readings each on a different property some of which are measured on the same ratio scale, we compare these readings as in the previous section. Finally we add the different indices derived for the different ratio scales and take their average for an overall index which should be no more than 1.10.

The foregoing discussion assumes that the measurements are independent of one another. If there is dependence among the factors it can be captured in part through $\alpha_{i}$ above. Measurements from several different ratio scales may be multiplied to form a single new ratio scale. This product may be compared with a similar product by forming the expression

which should be close to one.

$$
\frac{1}{2}\left[\frac{p_{1} p_{2} \cdots p_{n}}{q_{1} q_{2} \ldots q_{n}}+\frac{q_{1} q_{2} \cdots q_{n}}{p_{1} p_{2} \ldots p_{n}}\right]
$$

\section{ON THE POSSIBILITY OF ARROW'S IMPOSSIBILITY}

The economist Kenneth Arrow [3] in his work on the compatibility of a social utility function with individual utilities concluded that it is impossible that the ordering of alternatives by the group would always be consistent with the ordering of each of the participating individuals: His early work in proving this theorem is based on ordinal order requiring the assumption of transitivity of preferences. He and others have assumed that in fact their approach to the question coincides with how things actually are in the real world and in the world of logic and mathematics. This is not so. As a consequence of Arrow's work some people have concluded that dictatorships are the logical outcome and that democracy is unnatural. I will outline why this impossibility theorem can be misleading because of the assumptions it makes and because of the method it uses to arrive at the conclusion.

The way to analyze how individuals develop expectations about the compatibility of their rankings with that of a group to which they provide input is by assuming that each individual does carry 'out a complete ranking of the alternatives and compares it with the group ranking, originally proposed. Arrows method of ordinal ranking is not the only one, nor is it the most natural. Ranking can be made on a cardinal scale so that meaningful numbers are assigned to the alternatives rather than ordinals. There are two well established ways to create a cardinal rank order One of these is an ordering on a ratio scale which leads to the question of consistency, its measurement, allowing for inconsistency and deciding when a ranking is valid and when it is unjustified by the judgments. By allowing inconsistency for an individual or a group to adjust their judgments and incompatibility up to a certain tolerance level, it is possible 
that all individual's preferences would agree with group decision more often than Arrow's theory suggests. We are thus again freed of the idea that dictatorships are necessary.

Here we have the problem of the compatibility of $e^{T} A^{\circ} A_{i}^{T} e, i=1, \ldots, n$ where $A$ is the group derived scale matrix and $A_{i}$ are the individual matrices. The matrix $A$ may not coincide with $A_{i}$ but it can be sufficiently close and hence fall within a tolerable limit of each of them. In that case the individual could find the group ranking compatible with their individual rankings and need not dissent.

\section{REFERENCES}

1. Saaty, T.L., Fundamentals of Decision Making and Priority Theory with the Analytic Hierarchy Process, RWS Publications, 4922 Ellsworth Ave., Pittsburgh, PA, 15213 (1993).

2. Lyndon, R.C., "Groups and Geometry", London Mathematical Society Lecture Note Series 101, Cambridge University Press, Cambridge (1985). .

3. Arrow, K.J., Social Choice and Individual Values, (First edition, 1951). New York: Wiley (1963). 